\title{
Background assessment of the Nal(TI) crystals in the COSINE-100 experiment
}

\author{
Pushparaj Adhikari* \\ On behalf of the COSINE-100 collaboration \\ Department of Physics, Sejong University, Seoul 05006, Republic of Korea \\ E-mail: pushpaparticle@gmail.com
}

\begin{abstract}
The COSINE experiment is a joint effort between the KIMS-NaI and DM-Ice experiments to test the DAMA's claim on the WIMP discovery. It consists of $106 \mathrm{~kg}$ of $\mathrm{NaI}(\mathrm{Tl})$ crystals immersed in a 2 tons of liquid scintillator as an active veto. The construction of the first phase of the experiment, called COSINE-100, was completed in the summer of 2016. The physics run of the experiment has begun in September 2016 at the Yangyang Underground Laboratory in Korea. The current energy threshold is set at $2 \mathrm{keV}$ with $90 \%$ signal efficiency and the background level is 3 dru (Differential Rate Unit- count $/ \mathrm{keV} / \mathrm{kg} / \mathrm{day}$ ) at $2 \mathrm{keV}$. A background study and the performances of the COSINE-100 detector are presented.
\end{abstract}

35th International Cosmic Ray Conference - ICRC2017

10-20 July, 2017

Bexco, Busan, Korea

${ }^{*}$ Speaker. 


\section{Introduction}

Observations from the astronomical events including the velocities of stars and galexies, and gravitational-lensing measurements have inferred that about $27 \%$ of the Universe is made of dark matter. Rarely occuring interactions between supersymmetric Weakly Interacting Massive Particle (WIMP) comprising the Milky Way's dark matter halo and nuclei of normal matter may be measurable with a low-background detector in a deep underground laboratory.

DAMA group reported a positive annual modulation signal with a phase consistent with expectations from the Earth's motion relative to the Galactic rest frame [9]. DAMA measured the modulation amplitude in the singlesite event distribution at the energy range between 2-6 keV to be $0.0112 \pm 0.0012$ dru at $9.3 \sigma$, using 1.33 ton · year data with 1 dru background. With the phase of $144 \pm 7$ days and amplitude of $0.998 \pm 0.002$ years, its claimed signal is an indicative of WIMP interactions, while other experiments, including KIMS-CsI [5], reported null results. However, alternative interpretations of the DAMA results as being primarily due to WIMP-sodium nuclei interactions, which would be the case for low-mass WIMPs, cannot be ruled out. Therefore, it is necessary to try to reproduce the DAMA observations with crystal detectors of the same $\mathrm{NaI}(\mathrm{Tl})$ composition.

The COSINE experiment is a joint collaboration between the KIMS-NaI and the DM-Ice $\mathrm{NaI}(\mathrm{Tl})$ experiments to prove or refute DAMA's annual modulation result with $\mathrm{NaI}(\mathrm{Tl})$ crystals. The first phase of the experiment consisting of $106 \mathrm{~kg}$ of NaI(Tl) crystals and 2 tones of liquid scintillator as an active veto has started at the Yangyang underground laboratory in Korea.

\section{NaI crystals in COSINE-100}

The low background $\mathrm{NaI}(\mathrm{Tl})$ crystals were grown from highly purified powders. The eight $\mathrm{NaI}(\mathrm{Tl})$ crystals were produced by the Alpha Spectra(AS) Company. The initial purification of powder was carried out by the AS. The crystals have a cylindrical shape. There is a $12.7-\mathrm{mm}$-thick quartz-plate window at each end of the cylinder, with an optical grease between the crystal and the quartz windows. A 3 inch PMT is mounted at each end of the cylinder.

Crystals were produced by the AS from the NaI powders called AS-B for Crystal-1 and the same batch powder AS-C for Crystal-2, Crystal-5 and Crystal-8 . The details of the first two crystals are discussed in Ref. [8]. For the Crystal-3 and Crystal-4 production, AS developed additional background reduction methods, especially to reduce ${ }^{210} \mathrm{~Pb}$ contamination in the powder purification and crystal growing process. The batch of powder used for these crystals is called AS-WSII. For the Crystal- 6 and Crystal-7, AS tried to reduce the ${ }^{40} \mathrm{~K}$ and developed additional background reduction methods and produced a new batch of powder (called AS-WSIII). The details of the production dates of the crystals and delivery times are listed in Table. 1

Eight $\mathrm{NaI}$ crystals are put in an array of $4 \times 2$ in the core part of the detector. The total mass of the crystals is $106 \mathrm{~kg}$ and they are immersed in the LAB(Linear Alkyl Benzene) based liquid scintillator.

\subsection{Light yield}

Single photoelectron (SPE) spectra collected by each PMT are used to estimate the light 
Table 1: Specifications of the $\mathrm{NaI}(\mathrm{Tl})$ crystals. The "Powder" acronyms are AS-B (AS-C):Alpha Spectra purified powder, AS-WSII: Alpha Spectra WIMPScint-II grade powder and AS-WSIII: Alpha Spectra WIMPScint-III grade powder. The last two columns are the date of the crystals were grown and transported to $\mathrm{Y} 2 \mathrm{~L}$.

\begin{tabular}{lcccc} 
Crystal-ID & Mass $(\mathrm{kg})$ & Powder & T (Growth) & T (Y2L) \\
\hline Crystal-1 & 8.26 & AS-B & 2011.9 & 2013.9 \\
Crystal-2 & 9.15 & AS-C & 2013.4 & 2014.1 \\
Crystal-3 & 9.16 & AS-WSII & 2014.7 & 2014.12 \\
Crystal-4 & 18.01 & AS-WSII & 2015.10 & 2016.03 \\
Crystal-5 & 18.28 & AS-C & 2013.4 & 2016.03 \\
Crystal-6 & 12.5 & AS-WSIII & 2015.10 & 2016.02 \\
Crystal-7 & 12.5 & AS-WSIII & 2015.10 & 2016.02 \\
Crystal-8 & 18.28 & AS-C & 2013.4 & 2016.03
\end{tabular}

yield of each crystal. The light yields are measured by using $59.54 \mathrm{keV}$ gamma events from an ${ }^{241} \mathrm{Am}$ calibration source. The Light yield of each crystal is listed in the Table 2. The light yields of our crystal detectors are about 2 times larger than the DAMA crystal detectors [9]. The more light yields give better resolutions.

\subsection{Background activity of the crystals}

Background rates from internal radioactive contaminants in the $\mathrm{NaI}(\mathrm{Tl})$ crystals are measured in an R\&D setup [16] and also from the initial data of the COSINE-100 setup. A chain equilibrium is assumed for the interpretation of radioactivity measurements related to ${ }^{238} \mathrm{U}$ and ${ }^{232} \mathrm{Th}$, with the exception of ${ }^{210} \mathrm{~Pb}$. All the activities measured are listed in Table 2 .
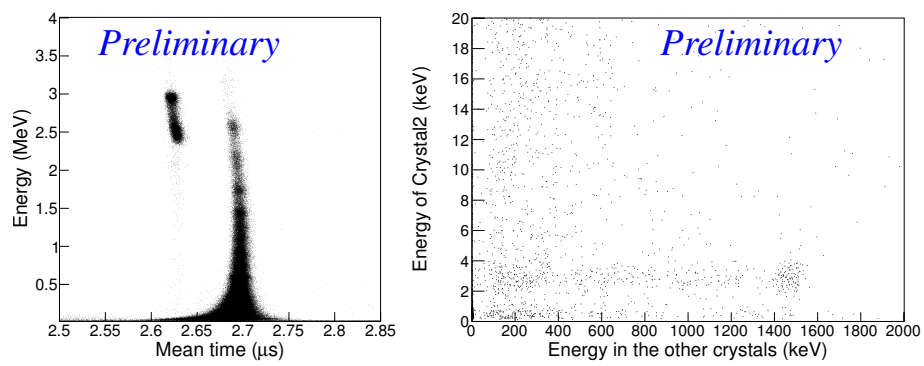

Figure 1: (Left)Meantime vs. Energy, the island with smaller mean time at higher energies is due to alphas and is clearly separated from gammas.(Right) Crystal-2 signal vs. accompanying crystals signal for multi-hit events.

\subsubsection{Alpha activities and ${ }^{210}$ Po background}

The pulse shape discrimination (PSD) based on amplitude weighted mean time of a waveform shows a good separation between alpha and gamma [8]. In the left plot of figure 1, the island with the lower mean time at the higher energies is due to alphas, and it is well separated from gamma events shown in the higher values. 
The ${ }^{238} \mathrm{U}$ and ${ }^{232} \mathrm{Th}$ contamination levels measured in the eight crystals are too low to account for the total observed $\alpha$ rates. This suggests that most of the $\alpha$ rate is due to decays of ${ }^{210} \mathrm{Po}$ (E $=5.4 \mathrm{MeV}$ ) nuclei originating from a ${ }^{222} \mathrm{Rn}$ exposure that occurred sometime during the powder and/or crystal processing stages $[8,16]$. The alpha rate for each crystal is listed in Table 2.

\subsection{2 ${ }^{40} \mathrm{~K}$ background}

In the setup, ${ }^{40} \mathrm{~K}$ decays are identified by coincidence signals between a $3 \mathrm{keV} X$-ray in one $\mathrm{NaI}(\mathrm{Tl})$ detector and a $1460 \mathrm{keV} \gamma$-ray in one of the other $\mathrm{NaI}(\mathrm{Tl})$ crystals. Figure 1 (right) shows a scatter-plot of the energy in Crystal-2 versus that in the other $\mathrm{NaI}(\mathrm{Tl})$ crystals for multi-hit coincidence events; the ${ }^{40} \mathrm{~K}$ signal is the island at $\sim 3 \mathrm{keV}$ in Crystal-2 signal and $\sim 1460 \mathrm{keV}$ in the other hit $\mathrm{NaI}(\mathrm{Tl})$ crystal. The ${ }^{40} \mathrm{~K}$ background level in each crystal is determined by comparing the measured coincidence rate with a Geant4-simulated efficiency using a method described in Ref. [8].

Table 2: Performances of crystals in the COSINE-100

\begin{tabular}{lccccc} 
Crystal & $\begin{array}{c}\text { Alpha Rate } \\
(\mathrm{mBq} / \mathrm{kg})\end{array}$ & $\begin{array}{c}{ }^{40} \mathrm{~K} \text { Activity } \\
(\mathrm{ppb})\end{array}$ & $\begin{array}{c}{ }^{238} \mathrm{U} \text { Activity } \\
(\mathrm{ppt})\end{array}$ & $\begin{array}{c}{ }^{232} \mathrm{Th} \\
(\mathrm{ppt})\end{array}$ & $\begin{array}{c}\text { Light Yield } \\
\text { (Photoelectrons/keV) }\end{array}$ \\
\hline Crystal-1 & $3.20 \pm 0.08$ & $43.4 \pm 13.7$ & $<0.02$ & $1.31 \pm 0.35$ & $14.88 \pm 1.49$ \\
Crystal-2 & $2.06 \pm 0.06$ & $82.7 \pm 12.7$ & $<0.12$ & $<0.63$ & $14.61 \pm 1.45$ \\
Crystal-3 & $0.76 \pm 0.02$ & $41.1 \pm 6.8$ & $<0.04$ & $0.44 \pm 0.19$ & $15.50 \pm 1.64$ \\
Crystal-4 & $0.74 \pm 0.02$ & $39.5 \pm 8.3$ & & $<0.3$ & $14.86 \pm 1.50$ \\
Crystal-5 & $2.06 \pm 0.05$ & $86.8 \pm 10.8$ & & $2.35 \pm 0.31$ & $7.33 \pm 0.70$ \\
Crystal-6 & $1.52 \pm 0.04$ & $12.2 \pm 4.5$ & $<0.018$ & $0.56 \pm 0.19$ & $14.56 \pm 1.45$ \\
Crystal-7 & $1.54 \pm 0.04$ & $18.8 \pm 5.3$ & & $<0.6$ & $13.97 \pm 1.41$ \\
Crystal-8 & $2.05 \pm 0.05$ & $56.15 \pm 8.1$ & & $<1.4$ & $3.50 \pm 0.33$
\end{tabular}

\section{Low energy noise rejection}
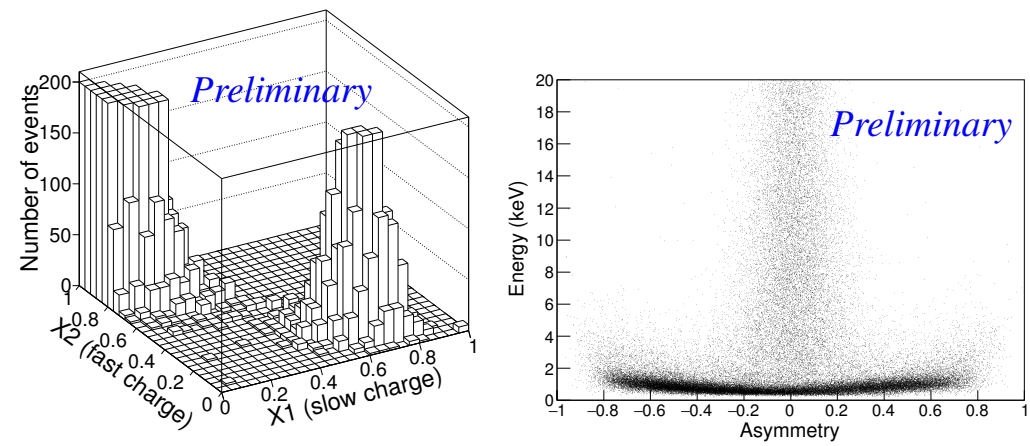

Figure 2: (left)Two-dimensional plots of fast and slow charges. PMT noise events have larger fast charges and signal events have larger slow charges.(right) Signal asymmetry determined from the left and right PMT charges. The middle vertical band centered near zero is beta/gamma region while a large spread below a few $\mathrm{keV}$ are PMT noise-like, or asymmetric charge events. 
The DAQ system collects a large amount of non-physics events that are mainly caused by the PMTs because of the relatively low hardware trigger threshold $(15 \mathrm{mV})$. These noise events might be due to radioactivity in the PMT glass or circuitry, the discharge of accumulated space charge in the PMT, PMT dark current, and afterpulses of large pulses, etc. Since PMT-generated noise signals mostly affect the lower energy region and can potentially mimic dark matter signals, these noise events have to be efficiently rejected by software selection criteria.

The DAMA group reported a signal selection criterion for efficiently removing PMT noise events from their $\mathrm{NaI}(\mathrm{Tl})$ detectors that exploits the fact that noise pulses are generally fast. The DAMA requirement places restrictions on the ratio of the fast charge $\mathrm{Q}_{\mathrm{f}}$ integrated in $[0,50 \mathrm{~ns}]$ time range, called X2, and the slow charge $\mathrm{Q}_{\mathrm{s}}$ in the $[100,600 \mathrm{~ns}$ ] time range, called $\mathrm{X} 1$ [1]. These parameters are defined as:

$$
\begin{aligned}
\mathrm{X} 1(\text { Slow Charge }) & =\frac{\mathrm{Q}_{\mathrm{s}}[100 \mathrm{~ns}, 600 \mathrm{~ns}]}{\mathrm{Q}_{\mathrm{tot}}[0 \mathrm{~ns}, 600 \mathrm{~ns}]} ; \\
\mathrm{X} 2(\text { Fast Charge }) & =\frac{\mathrm{Q}_{\mathrm{f}}[0 \mathrm{~ns}, 50 \mathrm{~ns}]}{\mathrm{Q}_{\mathrm{tot}}[0 \mathrm{~ns}, 600 \mathrm{~ns}]} .
\end{aligned}
$$

Although a large fraction of PMT noise events are removed by the DAMA requirement, some PMT noise-like events still remain. Therefore, we developed further requirements to remove these events. A charge asymmetry between the two PMTs was studied [8], and the asymmetry is defined as

$$
\text { Asymmetry }=\frac{\mathrm{Q} 1-\mathrm{Q} 2}{\mathrm{Q} 1+\mathrm{Q} 2},
$$

where Q1 and Q2 are the total charges measured by each PMTs.

We have started using boosted decision tree (BDT) techniques that account for correlations among the parameters. The multi-variate analysis using the charge ratio, asymmetry and additional parameters related with clusters of SPE shows improvement to separate noise events from gamma compared to application of linear straight cuts in sequence. Multiple site events as a beta/gamma sample using Compton scattering of ${ }^{60} \mathrm{Co}$ source are trained against single site events in data. Current energy threshold is set at $2 \mathrm{keV}$ with $90 \%$ signal efficiency using BDT technique. The BDT performance and the signal efficiency is as shown in figure 3 .
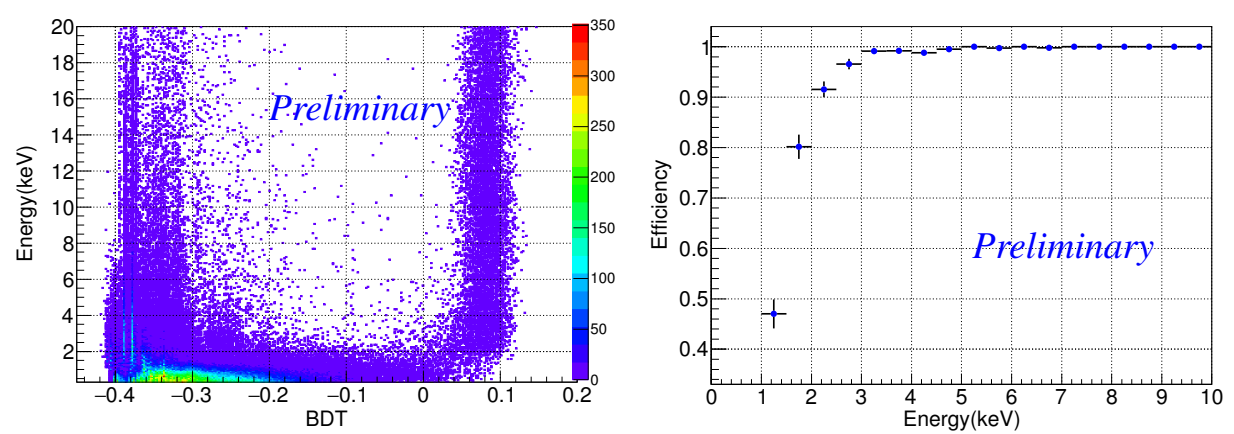

Figure 3: (Left)Two-dimensional plots of BDT variable and energy $(\mathrm{keV})$. The BDT variable is obtained from the training of beta sample from a ${ }^{60} \mathrm{Co}$ source using the noise rejection variables. (Right) Singnal efficiency of the BDT variable. 


\section{Liquid scintillator veto system}

There is a clear time correlation between LS and each crystal for the LS energies greater than $20 \mathrm{keV}$. After selecting LS energies above $20 \mathrm{keV}$, for instance, we have the clear coincidence spectrum from the Crystal-1 shown in figure 4 . And we confirm that we can tag the $3 \mathrm{keV} \mathrm{X-ray}$ from ${ }^{40} \mathrm{~K}$ with more than $70 \%$ efficiency. To get a firm characterization of the LS veto system, we measured the ${ }^{40} \mathrm{~K}$ activity levels of the eight crystals using LS coincidence events (i.e. Figure 4 (b) for Crystal-1) that are consistent with previous crystal-crystal coincidence measurements.

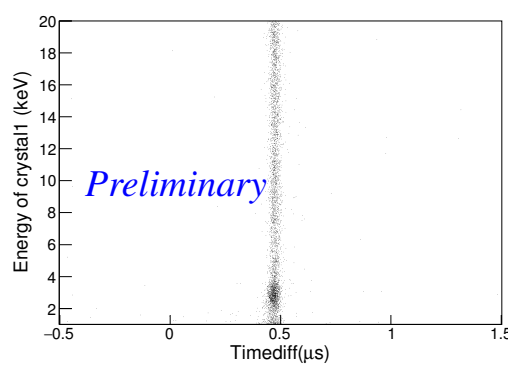

(a)

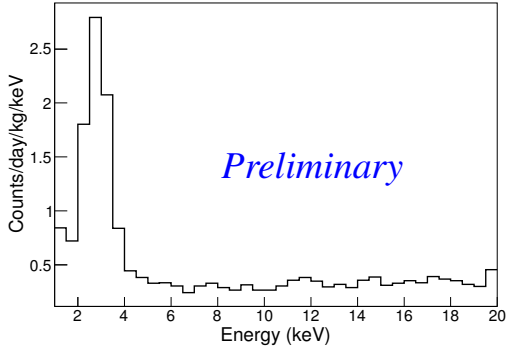

(b)

Figure 4: With the application of an LS energy greater than $20 \mathrm{keV}$ requirement we measure: (left) the time difference between the maximum LS pulse-height time and crystal-1 trigger time versus Crystal-1 energy(keV). $20 \mathrm{keV}$ LS energy threshold has been applied to see the coincident events. (right) Vetoed events (coincidence events) in the low energy region of Crystal-1. The $3 \mathrm{keV}$ peak of ${ }^{40} \mathrm{~K}$ is vetoed with $70 \%$ efficiency.

\section{Background summary}

The high-energy spectrum from $100 \mathrm{keV}$ to $2 \mathrm{MeV}$ shows several gamma peaks. A $1460 \mathrm{keV}$ $\gamma$-ray from ${ }^{40} \mathrm{~K}$ and $\gamma$ lines from daughter nuclei in the ${ }^{238} \mathrm{U}$ and ${ }^{232} \mathrm{Th}$ decay chains are main contributors. The overall background level is reduced by as much as $80 \%$ by requiring single-hit crystal events with the application of LS tagging threshold. Using a Geant-4 simulation, we have studied the background contribution from various isotopes. The modeling of the single hit energy spectrum of Crystal-7 is shown in figure. 5. The internal background components ${ }^{40} \mathrm{~K}$, and ${ }^{210} \mathrm{~Pb}$, and external background contributions from detector PMTs and also ${ }^{210} \mathrm{~Pb}$ from crystal surface and crystal encapsulation copper surface are the main background source in the region of interest at energies between 2-20 keV. Current discrepancy between data and MC is about 20\%. Further fine tuning of simulation is necessary to reach better data-MC agreement.

Figure 6 shows a comparison of background levels in Crystal-1, Crystal-3 and Crystal-7. A factor of four reduction in the ${ }^{210} \mathrm{~Pb}$ contamination level is observed in Crystal-3 compared to that of Crystal-1. Crystal-7 shows a lower ${ }^{210} \mathrm{~Pb}$ contamination compared to Crystal-1, and its ${ }^{40} \mathrm{~K}$ contamination level is $15 \mathrm{ppb}$. Below $6 \mathrm{keV},{ }^{40} \mathrm{~K} \mathrm{X}$-rays and cosmogenic activations of I, Te, and ${ }^{22} \mathrm{Na}$ isotopes are the main contributors to the remaining background. The cosmogenically activated backgrounds will diminish significanly after a year in the deep underground place at the Y2L. 

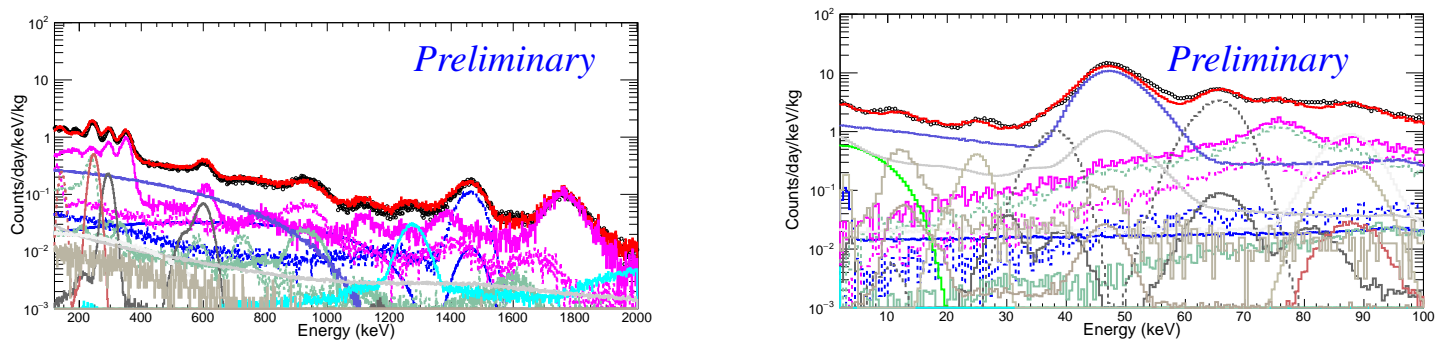

Figure 5: (left) The high energy (100keV-2000keV) measured background spectrum (black circle) is fitted with simulated background spectra for single hit events. The solid red line is the sum of simulated backgrounds. (right) The same spectrum as left for low energy (below 100keV).
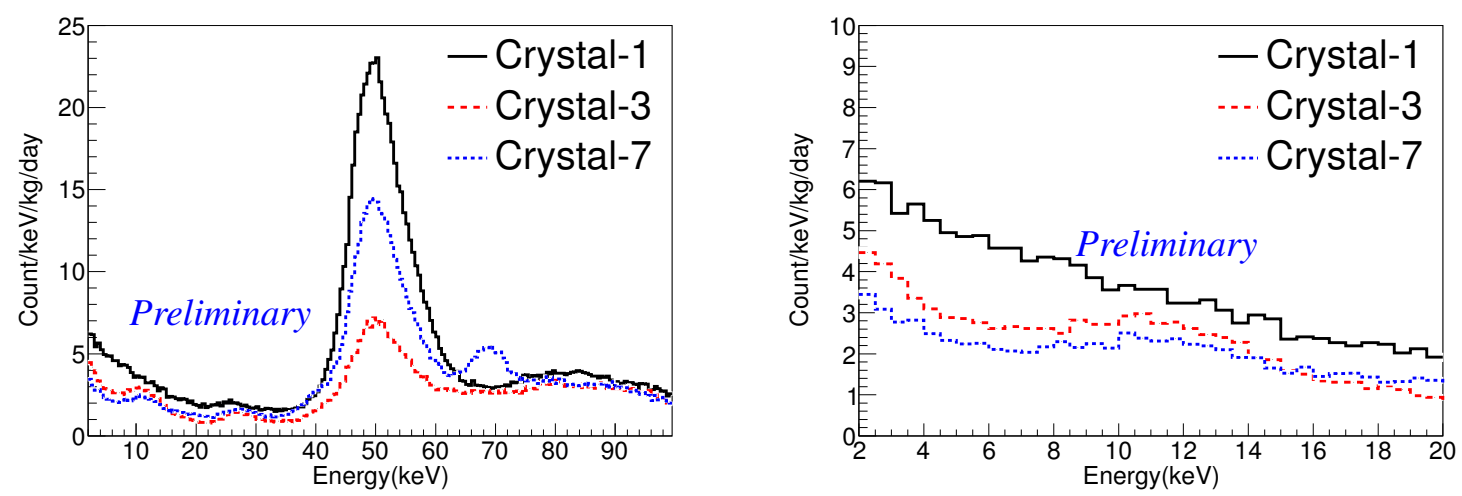

Figure 6: (Left) Spectra of three crystals from different powder samples. The peak near $50 \mathrm{keV}$ reflects the ${ }^{210} \mathrm{~Pb}$ contamination level in each crystal. Crystal-7 has been at underground for less than one year and, so, it contains cosmogenic peaks (e.g., ${ }^{125} \mathrm{I}$ ) (right) A zoomed-in plot to show the spectra for energies below $20 \mathrm{keV}$.

\section{Summary and outlook}

The goal of the COSINE-100 experiment is to confirm or refute the DAMA's annual modulation signature. The detector consists of $106 \mathrm{~kg}$ of NaI(Tl) crystals immersed in 2 tones of liquid scintillator as an active veto. The detector has been taking data since September 30, 2016 and the fraction of physics data is more than $90 \%$. Initial data show a sound performance in terms of light yield (15 photoelectron/keV) and the background level. The ${ }^{40} \mathrm{~K}$ background level is similar with DAMA (12 ppb in Crystal-6) and the ${ }^{238} \mathrm{U}$ and ${ }^{232} \mathrm{Th}$ activity is less than $1 \mathrm{ppt}$. Current energy threshold of the detector is set at $2 \mathrm{keV}$ with $90 \%$ signal efficiency. More precise signal selection is ongoing to reduce the energy threshold to below $1 \mathrm{keV}$.

\section{Acknowledgements}

We thank the Korea Hydro and Nuclear Power (KHNP) Company for providing the underground laboratory space at Yangyang. We also acknowlege the following supports : the Institute 
for Basic Science (IBS) under project code IBS-R016-A1, Republic of Korea; the Alfred P. Sloan Foundation Fellowship, NSF Grants No. PHY-1151795 and PHY-1457995, WIPAC, the Wisconsin Alumni Research Foundation, and Yale University, United States; STFC grant ST/N000277/1, United Kingdom; CNPq, Brazil.

\section{References}

[1] R. Bernabei et al. (DAMA/LIBRA Collaboration), New results from DAMA/LIBRA, Eur. Phys. J. C 67 (2010) 39 .

[2] E, Aoruke et al. (XENON100 Collaboration), Dark Matter Results from 225 Live Days of XENON100 Data, Phys. Rev. Lett. 109 (2012) 181301.

[3] D. S. Akerib et al. (LUX Collaboration), First results from the LUX dark matter experiment at the Stanford Underground Research Facility, Phys. Rev. Lett. 112 (2014) 091303.

[4] R. Agness et al. (CDMS Collaboration), Search for Low-Mass Weakly INteracting Massive Particle Using Voltage-assited Calorimetric Ionization Detection in teh SuperCDMS Experiment, Phys. Rev. Lett. 112 (2014) 041302.

[5] H. S. Lee et al. (KIMS Collaboration), First limit on WIMP cross section with low background CsI(Tl) crystal detector, Phys. Lett. B $\mathbf{6 3 3}$ (2006) 201.

[6] H. S. Lee et al. (KIMS Collaboration), Limits on Interactions between weakly interacting massive particles and Nucleons Obtained with CSI(Tl) Crystal Detectors, Phys. Rev. Lett. 99 (2007) 091301.

[7] S. C. Kim et al. (KIMS Collaboration), New limits on interactions between weakly interacting massive particles and nucleons obtained with CsI(Tl) crystal detectors, Phys. Rev. Lett. 108 (2012) 181301.

[8] K. W. Kim et al., Test on NaI(Tl) crystals for WIMP search at the Yangyang Underground Laboratory, Astropart. Phys. 62 (2015) 249.

[9] R. Bernabei et al., The DAMA/LIBRA apparatus, Nucl. Instrum. Methods Phys. Res., Sect. A 592 (2008) 3.

[10] G. Gerbier et al., Pulse shape discrimination and dark matter search with NaI(Tl) scintillator, Astropart. Phys. 11 (1999) 287.

[11] Y. D. Kim et al., Inhibition of 137Cs contamination in cesium iodide, Nucl.Instrum.Meth.A. 552 (2005) 456.

[12] H. S. Lee et al., Development of low background CsI(Tl) crystals for WIMP search, Nucl.Instrum.Meth.A. 571 (2005) 644.

[13] H. S. Lee et al., Neutron calibration facility with an Am-Be source for pulse shape discrimination measurement of CSI(Tl) crystals, JINST 9 (2014) P11015.

[14] H. S. Lee et al., Pulse-shape discrimination between electron and nuclear recoils in a NaI(Tl) crystal, JHEP (2015)

[15] J. Amare et al., Cosmogenic radionuclide production in NaI(Tl) crystals, J. Cosmol. Astropart. Phys. 1502 (2015) 046.

[16] P. Adhikari et al. (KIMS Collaboration), Understanding internal backgrounds of NaI(Tl) crystals toward a $200 \mathrm{~kg}$ array of the KIMS-NaI experiment, Eur. Phys. J. C 76 (2016) 185. 